\title{
The 15th International Symposium on High Pressure Low Temperature Plasma Chemistry (HAKONE XV)
}

The 15th International Symposium on High Pressure Low Temperature Plasma Chemistry (HAKONE XV) was held in Brno, Czech Republic, from September 11th to 16th 2016. The conference was jointly organized by the Masaryk University (CEPLANT, Department of Physical Electronics at the Faculty of Science) and The Union of Czech Mathematicians and Physicists Brno, Czech Republic. With HAKONE XV a series of successful conferences devoted to the fundamentals and applications of non-thermal plasmas at elevated pressures was continued. Over 120 regular attendees, 8 accompanying persons and guests from 26 different countries participated and submitted 118 contributions in 9 different topics.

HAKONE is a bi-annual symposium bringing together scientists and engineers working on subjects in the basic research and technology of high pressure (typically 1 bar) and low temperature plasma chemistry. The traditional HAKONE topics are dealing with fundamental aspects of gas discharge physics and chemistry at elevated pressures, surface processing technology, ozone generation and environmental applications and were represented strongly also in Brno. However, gradually in time the scope of HAKONE interests has been extended to newly emerging and innovative fields of research and development, like biomedical plasma applications, alternative materials, fuels and other interdisciplinary plasma topics. The invited lectures covered the following topics:

- Plasma-based $\mathrm{CO}_{2}$ conversion: A combined modeling and experimental study by Annemie Bogaerts (Belgium).

- Optical emission diagnostics for nanosecond microdischarges at atmospheric pressure by Yi-Kang Pu (China).

- Measurement of electric field in atmospheric pressure discharges using Stark polarization spectroscopy by Bratislav Obradović (Serbia).

- Non-thermal plasma combined with catalysts for environmental technology by Akira Mizuno (Japan).

- Confined atmospheric plasma sources for activating gases, liquids and tissue by Mark J. Kushner (USA).

- Lateral patterns in glow-like barrier discharges by Lars Stollenwerk (Germany).

It was a pleasure to welcome also the community of joint one-day COST action Workshop TD1208 on Electrical Discharges with Liquids for Future Applications. This workshop enriched the scope of the conference for following invited lecture:

- Plasmas interacting with liquids: Energy transfer and chemistry by Antoine Rousseau (France).

The HAKONE XV conference was dedicated to the memory of Dr. Ulrich Kogelschatz, who passed away in June 2016. Ulrich Kogelschatz was an outstanding scientist and good friend to many of us, and one of the founders of the HAKONE symposium.

The ISC continued the collaboration with the European Physical Journal - Applied Physics and prepared this special issue of the journal devoted to the conference. All contributed papers of the HAKONE XV symposium are available in the conference proceedings distributed electronically to all participants. They are also publicly available here: https://munispace.muni.cz/book?id=836.

Finally we would like to thank all participants of HAKONE XV and invite the readers of the journal to the next HAKONE symposium, which will be held in fall 2018 in Beijing, China.

Tomáš Hoder

Vice-chair of the Local Organising Committee

Mirko Černák

Chair of the International Scientific Committee 